\title{
Impact of Public Sector Financial Audit: A Case Study on Police Department of Abu Dhabi, UAE
}

\author{
Hmoud Shelaiweeh Ali Aljauaan Alshamsi ${ }^{1}$, \\ Joseph Adaikalam², Asif Mahbub Karim ${ }^{3}$ \\ ${ }^{1}$ PhD Research Fellow, Binary University of Management \& Entrepreneurship, Malaysia \\ ${ }^{2}$ Founder and Executive Chairman, Binary University of Management \& Entrepreneurship, Malaysia \\ ${ }^{3}$ Dean \& Associate Professor, Binary Graduate School, Binary University of Management \& Entrepreneurship, Malaysia \\ ${ }^{3}$ E-mail: drasifmkarim@gmail.com (Corresponding author)
}

Abstract

Public sector audits involve checking the financial issues of state-owned entities that have been conducted in the best interest of the public, and to determine whether standard procedures have been followed to adhere to the procedures required to promote transparency and good governance (such as public sector procurement rules). Public sector audits therefore go a step further than the financial audits of nongovernmental companies that focus primarily on the reliability of financial statements. The specific objective of this study is to investigate the public sector financial audit effectiveness in the Abu Dhabi Police (ADP), UAE. Other objectives are, to investigate impact of infrastructure for financial audit in the Police Department to improve performance, to investigate the competency of the public sector financial auditors in the ADP, UAE. Some of the other factors that can impact the efficiency of the governmental audit process are the independence and motivation of the institute which can conduct the work of the audit without any department intervention for audit work. The freedom of internal audit and assurance for the objectivity institute is both in service and in consultation. The first objective of the study, it is seen that the effectiveness of the public sector financial audit will be more appropriate if there is a scope of joint audit and both local and global reputed audit firms are involved. The research has focused the need of such need of the hour independent acceptability to be at par with leading nation globally. Secondly, impact of the competency of the public sector financial audit was observed to be very ineffective due to the fact it is just a routine check, rather the competency would have been improved if in addition surprise public sector financial audit was determined and put into place.

Key words Public Sector, Financial Audit, Police, UAE

Received: 03 Feb $2020 \quad$ (C) The Authors 2020

Revised: 14 Feb 2020 Published by Human Resource Management Academic Research Society (www.hrmars.com)

Accepted: 24 Feb 2020 This article is published under the Creative Commons Attribution (CC BY 4.0) license. Anyone may Published 2020 reproduce, distribute, translate and create derivative works of this article (for both commercial and non-commercial purposes), subject to full attribution to the original publication and authors. The full terms of this license may be seen at: http://creativecommons.org/licences/by/4.0/legalcode

\section{Introduction}

The audit is an examination conducted by a competent professional and independent opinion to express a reasoned opinion on the regularity and the fairness of the statement of financial position and information annexes to the annual accounts of an enterprise "(Ordre des Experts-Compatibles (OEC). According to the National Company of Auditors (CNCC) an audit consists of examining, by sampling the evidence justifying the data contained in the accounts. It consists also to assess the accounting principles followed and the significant estimates used to draw up the accounts and to appreciate their overall presentation. At the international level, in the IFAC standards: "The financial audit has purpose of allowing 
the auditor to express an opinion according to the financial statements have been prepared in all their aspects significant, in accordance with a defined accounting framework." The audit can be internal to the company, which leads us to say that there are two types of audit, the external audit and the internal audit: Internal audit and External audit.

The following definition given by the French Institute of Auditors and Internal Controllers (IFACI) reflects the evolution and reflects the current conception of internal audit: "Internal audit is the periodic review of the instruments available to a management to control and manage the business. This activity is carried out by a department dependent on the management of the company and independent of other services ".

The purpose of the internal auditors' policy is to promote the ethical culture in the practice of the internal auditing profession. Internal audit is an independent and objective activity of assurance and advice, aimed at improving the efficiency and efficiency of the organization. It assists the organization in pursuing its objectives through a systematic professional approach that generates value added as it is aimed at assessing and improving risk management, control and governance processes. The presence of internal control systems serves only as a "watch dog" established by regulators and policy makers to promote the motivation, due process and accountability (Jill \& Humes, 2014) to increase public confidence in state agencies.

Abu Dhabi Police Force was established on June 01, 1956, and was based in "Naïf Fort," as its Headquarters, until the year 1973 when it was moved to the current location. "Naif Fort" was transformed, later, into one of Dubai Police Stations. Abu Dhabi Police is one of the most challenging agencies in all UAE public sector organizations.

Also, the police are one of the most diverse forces and need to be very attentive, active and effective and object oriented. Abu Dhabi police therefore consider that their staff should have the skills and confidence to handle crisis situations (Gru-Gumbao et al., 2002) who say that equipping human resources with the required level of professionalism is the ability to face these challenges.

Increasingly, good performance in the public sector is not seen as simply achieving what was planned. The behavior of the aforementioned business issue is always public; People expect that their tax money and authority given to governments will be exercised with discretion, possibility, integrity and justice. Said et al (2016) argued that the ability to adapt is a key feature of a successful public organization: As future events unfold, firms need to continue to provide and continue learning and learning. If there would be no control and audit of the public officials, there is an impending risk that fraud, corruption and other mismanagement would increase (Axelsen et al., 2017).

\section{Literature review}

The Internal Audit Institute defines a broad definition of Internal Audit through the International Standards of Internal Audit, adopted internationally as the Internal Audit Standard: A self-governing and purposeful activity ensures the degree to which an Institute operates, to develop its activities and to provide additional benefits. This is a guide to contributing to the issues. The role of internal audit is to propose the individual or team to achieve its objectives, to evaluate the processes of controlling and managing the organization's risk management through managerial and systematic approaches, and to come up with proposals to consolidate their effectiveness.

Internal auditors which are the main concern of this study furthermore the key representative of open workplaces, are anticipated that would work autonomously and dispassionately to upgrade high calibre of public management, accomplish great internal audit framework, maintain a strategic distance from defilement, guarantee great corporate administration framework, advance responsibility and more prominent straightforwardness (Yuan et al., 2007).

Freedom and objectivity are closely related at the time of rendering the neutral process, where conditions that may threaten the compromise of objectivity and irrelevant values are released (Internal Audit Institute, I). In fact, there should be a fair enough amount of freedom from those responsible for the audit to be monitored so that it can both manage its work without any hassle and be able to do so (Mansour, 2017). 
This is very important in the implementation of financial audits because auditors can access any document related to the audit work without fear and fear. It will provide auditors with reports on the audit process and credible professional judgment in order to achieve the order issued by their government. If auditors do not appear to exercise the same capabilities as a member of the client management or an employee, the independence of the auditor shall not be deemed to be impaired. There is confusion about the role of financial internal auditing because internal auditors are part of the administration team and at the same time can independently evaluate the effectiveness and efficiency of management. In recent years, however, sensitive attention has been paid to issues associated with the independence and purpose of internal audit. This can affect the ability of internal auditing although internal auditors are accused of maintaining the best comfort to their employer, but they may express disregard for counter management regardless of the punishment in management (Zureigat, 2011).

Tricker \& Tricker (2015) in his research examined the literature on the effectiveness of internal audit in corporate governance and the scope of future research. They also focus on the relationship between internal audit and other foundations of governance (such as external auditors, audit committees and management), they also evaluate the above literature on the quality of internal audits, including objectivity and independence. The reviewer (Coram et al., 2008) found that if the internal management function of the corporate administration depends on the quality of the external auditor, the determinant of its decision, the extent and nature of its work, the external auditor and other aspects of the external audit were analyzed. On the other hand (Fan \& Wang, 2005) discussed the importance of internal audit groups in corporations. A member of the Chicago chapter of the IIA, along with its current entities, conducted a survey to establish which procedures, preferred practices and gaps related to internal audit, independent of scope, and Sarbanes-Oxley compliance audit were established.

Accordingly, it is crucial to have forceful internal auditor unit as a major aspect of present day management framework out in the public division in government. In corporate financial internal auditor issue has gotten expanding consideration as of late, because of various reasons. To specify some of them, internal auditor connections to the inward controlling hazard management framework; enhance organization proficiency and effectiveness and viability through giving valuable feedback and recommendations about relations status; minimize data asymmetry amid basic management; serves as an necessary internal confirmation in the trade and budgetary reporting procedure of enterprises (Güvemli \& Yıldız, 2010).

Some of the other factors that can impact the efficiency of the governmental audit process are the independence and motivation of the institute which can conduct the work of the audit without any department intervention for audit work. The freedom of internal audit and assurance for the objectivity institute is both in service and in consultation. Freedom and objectivity are closely related when rendering the audit process where situations may threaten to compromise the unrealistic and irrelevant values (Institute of Internal Auditors, 1999). In fact, the audit task should have enough degree of freedom from those it is required to audit so that it can both conduct its work without any disturbing and be seen to be able to do so (Institute of Internal Auditors, 2001). This is very important in the implementation of financial audits because auditors can access any document related to the audit work without fear and fear. It will provide auditors with reports on the audit process and credible professional judgment in order to achieve the order issued by their government. If auditors do not appear to exercise the same capabilities as a member of the client management or an employee, the independence of the auditor shall not be deemed to be impaired. There is a misconception about the role of financial internal auditing because internal auditors are part of the administration team and at the same time can independently evaluate the effectiveness and efficiency of management.

In recent years, however, sensitive attention has been paid to issues associated with the independence and purpose of internal audit. This can affect the ability of internal auditing although internal auditors are accused of maintaining the best comfort to their employer, but they may express disregard for counter management regardless of the punishment in management (Zureigat, 2011).

Gramling et al. (2004) in his research examined the literature on the effectiveness of internal audit in corporate governance and the scope of future research. They focused on the relationship between internal audit and other foundations of governance (i.e. external auditors, audit committees and management). 
They also evaluated the above literature on the quality of internal audit with purpose and uniqueness. The reviewer (Coram et al., 2008) found that if the internal management function of the corporate administration depends on the quality of the external auditor, the determinant of its decision, the extent and nature of its work, the external auditor and other aspects of the external audit were analyzed.

\section{Conceptual framework}

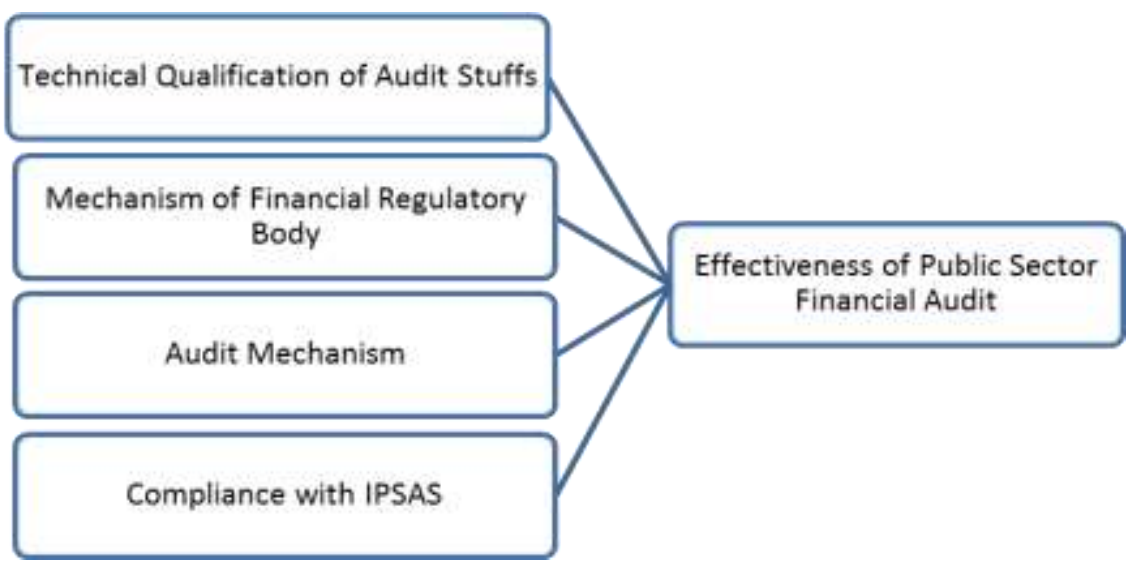

Source: Authors

\section{Methodology of research and Findings \& Data Analysis}

\subsection{Data of questionnaire Administered from Managers}

Demographic part:

This section of the study aimed at establishing the number of respondent's departments. The four departments of Police of United Arab Emirates are involved in budget process are given below.

1. Accounting

2. Management

3. Economics

4. others

A total of 150 questionnaires were distributed and all were filled and returned giving a response rate of $100 \%$ from the managers sector. This was sufficient for the study.

Level of education

\begin{tabular}{|c|l|c|c|c|c|}
\hline \multirow{3}{*}{ Valid } & & Frequency & Percent & Valid Percent & Cumulative Percent \\
\cline { 2 - 6 } & TVT certificate & 30 & 20.0 & 20.0 & 20.0 \\
\cline { 2 - 6 } & Diploma & 50 & 33.3 & 33.3 & 53.3 \\
\cline { 2 - 6 } & Bachelor's degree & 39 & 26.0 & 26.0 & 79.3 \\
\cline { 2 - 6 } & Master's degree & 22 & 14.7 & 14.7 & 94.0 \\
\cline { 2 - 6 } & Others & 9 & 6.0 & 6.0 & 100.0 \\
\cline { 2 - 6 } & Total & 150 & 100.0 & 100.0 & \\
\hline
\end{tabular}

Internal auditors can effectively identify and report any noncompliance activities with my office's/sector's policies and procedures.

\begin{tabular}{|c|l|c|c|c|c|}
\hline & & Frequency & Percent & Valid Percent & Cumulative Percent \\
\hline \multirow{3}{*}{ Valid } & strongly disagree & 6 & 4.0 & 4.0 & 4.0 \\
\cline { 2 - 6 } & Disagree & 12 & 8.0 & 8.0 & 12.0 \\
\cline { 2 - 6 } & Neutral & 27 & 18.0 & 18.0 & 30.0 \\
\cline { 2 - 6 } & Agree & 25 & 16.7 & 16.7 & 46.7 \\
\cline { 2 - 6 } & strongly agree & 80 & 53.3 & 53.3 & 100.0 \\
\cline { 2 - 6 } & Total & 150 & 100.0 & 100.0 & \\
\hline
\end{tabular}


Internal auditors provide useful recommendations and constructive criticisms on noncompliance activities or control systems of the office/sector.

\begin{tabular}{|l|l|c|c|c|c|}
\hline & & Frequency & Percent & Valid Percent & Cumulative Percent \\
\hline \multirow{3}{*}{ Valid } & strongly disagree & 1 & .7 & .7 & .7 \\
\cline { 2 - 6 } & Disagree & 2 & 1.3 & 1.3 & 2.0 \\
\cline { 2 - 6 } & Neutral & 11 & 7.3 & 7.3 & 9.3 \\
\cline { 2 - 6 } & Agree & 33 & 22.0 & 22.0 & 31.3 \\
\cline { 2 - 6 } & strongly agree & 103 & 68.7 & 68.7 & 100.0 \\
\cline { 2 - 6 } & Total & 150 & 100.0 & 100.0 & \\
\hline
\end{tabular}

I use the recommendations, criticisms and information provided by internal auditor for decision making.

\begin{tabular}{|l|l|c|c|c|c|}
\hline & & Frequency & Percent & Valid Percent & Cumulative Percent \\
\hline \multirow{4}{*}{ Valid } & Neutral & 2 & 1.3 & 1.3 & 1.3 \\
\cline { 2 - 6 } & Agree & 62 & 41.3 & 41.3 & 42.7 \\
\cline { 2 - 6 } & strongly agree & 86 & 57.3 & 57.3 & 100.0 \\
\cline { 2 - 6 } & Total & 150 & 100.0 & 100.0 & \\
\hline
\end{tabular}

My office/sector has put its confidence on internal audit staffs, because they may face any problems(non-compliance activities).

\begin{tabular}{|l|l|c|c|c|c|}
\hline & & Frequency & Percent & Valid Percent & Cumulative Percent \\
\hline \multirow{3}{*}{ Valid } & Neutral & 6 & 4.0 & 4.0 & 4.0 \\
\cline { 2 - 6 } & Agree & 79 & 52.7 & 52.7 & 56.7 \\
\cline { 2 - 6 } & strongly agree & 65 & 43.3 & 43.3 & 100.0 \\
\cline { 2 - 6 } & Total & 150 & 100.0 & 100.0 & \\
\hline
\end{tabular}

The noncompliance reports provided internal auditors are reliable and significant to my organization/sector.

\begin{tabular}{|c|l|c|c|c|c|}
\hline \multirow{3}{*}{ Valid } & & Frequency & Percent & Valid Percent & Cumulative Percent \\
\cline { 2 - 6 } & Disagree & 1 & .7 & .7 & .7 \\
\cline { 2 - 6 } & Neutral & 6 & 4.0 & 4.0 & 4.7 \\
\cline { 2 - 6 } & Agree & 107 & 71.3 & 71.3 & 76.0 \\
\cline { 2 - 6 } & strongly agree & 36 & 24.0 & 24.0 & 100.0 \\
\cline { 2 - 6 } & Total & 150 & 100.0 & 100.0 & \\
\hline
\end{tabular}

The internal auditors have confidence to issue audit report because they are capable to determine the nature and frequency of noncompliance activities.

\begin{tabular}{|l|l|c|c|c|c|}
\hline \multirow{3}{*}{ Valid } & & Frequency & Percent & Valid Percent & Cumulative Percent \\
\cline { 2 - 6 } & Neutral & 15 & 10.0 & 10.0 & 10.0 \\
\cline { 2 - 6 } & Agree & 84 & 56.0 & 56.0 & 66.0 \\
\cline { 2 - 6 } & strongly agree & 51 & 34.0 & 34.0 & 100.0 \\
\cline { 2 - 6 } & Total & 150 & 100.0 & 100.0 & \\
\hline
\end{tabular}

The number of complaints (doubts) about the internal auditors finding(report) is very low because their report is correct and reasonable.

\begin{tabular}{|c|c|c|c|c|c|}
\hline & & Frequency & Percent & Valid Percent & Cumulative Percent \\
\hline \multirow[t]{2}{*}{ Valid } & Neutral & 24 & 16.0 & 16.0 & 16.0 \\
\hline & Agree & 77 & 51.3 & 51.3 & 67.3 \\
\hline
\end{tabular}


International Journal of Academic Research in Accounting, Finance and Management Sciences

Vol. 10 (1), pp. 25-40, @ 2020 HRMARS (www.hrmars.com)

\begin{tabular}{|l|l|c|c|c|c|}
\hline \multirow{2}{*}{} & strongly agree & 49 & 32.7 & 32.7 & 100.0 \\
\cline { 2 - 6 } & Total & 150 & 100.0 & 100.0 & \\
\hline
\end{tabular}

Internal audit ensures the economical, effective and efficient use of resources in my office/sector.

\begin{tabular}{|c|l|c|c|c|c|}
\hline \multirow{3}{*}{ Valid } & & Frequency & Percent & Valid Percent & Cumulative Percent \\
\cline { 2 - 6 } & Disagree & 1 & .7 & .7 & .7 \\
\cline { 2 - 6 } & Neutral & 26 & 17.3 & 17.3 & 18.0 \\
\cline { 2 - 6 } & Agree & 67 & 44.7 & 44.7 & 62.7 \\
\cline { 2 - 6 } & strongly agree & 56 & 37.3 & 37.3 & 100.0 \\
\cline { 2 - 6 } & Total & 150 & 100.0 & 100.0 & \\
\hline
\end{tabular}

In my office/sector internal audit ensures activity performed is compliance with established policies, procedures, laws and regulations.

\begin{tabular}{|l|l|c|c|c|c|}
\hline & & Frequency & Percent & Valid Percent & Cumulative Percent \\
\hline \multirow{3}{*}{ Valid } & Disagree & 5 & 3.3 & 3.3 & 3.3 \\
\cline { 2 - 6 } & Neutral & 26 & 17.3 & 17.3 & 20.7 \\
\cline { 2 - 6 } & Agree & 58 & 38.7 & 38.7 & 59.3 \\
\cline { 2 - 6 } & strongly agree & 61 & 40.7 & 40.7 & 100.0 \\
\cline { 2 - 6 } & Total & 150 & 100.0 & 100.0 & \\
\hline
\end{tabular}

The recommendation of internal audit department provides practical, cost-benefit solutions for correcting the problems that were found.

\begin{tabular}{|l|l|c|c|c|c|}
\hline & & Frequency & Percent & Valid Percent & Cumulative Percent \\
\hline \multirow{3}{*}{ Valid } & Disagree & 3 & 2.0 & 2.0 & 2.0 \\
\cline { 2 - 6 } & Neutral & 28 & 18.7 & 18.7 & 20.7 \\
\cline { 2 - 6 } & Agree & 73 & 48.7 & 48.7 & 69.3 \\
\cline { 2 - 6 } & strongly agree & 46 & 30.7 & 30.7 & 100.0 \\
\cline { 2 - 6 } & Total & 150 & 100.0 & 100.0 & \\
\hline
\end{tabular}

Internal auditors have the experience and expertise to address corporate risk management problems within the organization.

\begin{tabular}{|c|l|c|c|c|c|}
\hline & & Frequency & Percent & Valid Percent & Cumulative Percent \\
\hline \multirow{3}{*}{ Valid } & Disagree & 6 & 4.0 & 4.0 & 4.0 \\
\cline { 2 - 6 } & Neutral & 22 & 14.7 & 14.7 & 18.7 \\
\cline { 2 - 6 } & Agree & 71 & 47.3 & 47.3 & 66.0 \\
\cline { 2 - 6 } & strongly agree & 51 & 34.0 & 34.0 & 100.0 \\
\cline { 2 - 6 } & Total & 150 & 100.0 & 100.0 & \\
\hline
\end{tabular}

The existing role that the internal audit is playing sufficient enough to address the very purpose for which it is established.

\begin{tabular}{|l|l|c|c|c|c|}
\hline \multirow{3}{*}{ Valid } & & Frequency & Percent & Valid Percent & Cumulative Percent \\
\cline { 2 - 6 } & Disagree & 7 & 4.7 & 4.7 & 4.7 \\
\cline { 2 - 6 } & Neutral & 29 & 19.3 & 19.3 & 24.0 \\
\cline { 2 - 6 } & Agree & 57 & 38.0 & 38.0 & 62.0 \\
\cline { 2 - 6 } & strongly agree & 57 & 38.0 & 38.0 & 100.0 \\
\cline { 2 - 6 } & Total & 150 & 100.0 & 100.0 & \\
\hline
\end{tabular}


Internal auditors of your company are capable of carrying out internal control function under current corporate governance rules.

\begin{tabular}{|l|l|c|c|c|c|}
\hline & & Frequency & Percent & Valid Percent & Cumulative Percent \\
\hline \multirow{3}{*}{ Valid } & Disagree & 4 & 2.7 & 2.7 & 2.7 \\
\cline { 2 - 6 } & Neutral & 33 & 22.0 & 22.0 & 24.7 \\
\cline { 2 - 6 } & Agree & 74 & 49.3 & 49.3 & 74.0 \\
\cline { 2 - 6 } & Strongly agree & 39 & 26.0 & 26.0 & 100.0 \\
\cline { 2 - 6 } & Total & 150 & 100.0 & 100.0 & \\
\hline
\end{tabular}

The existence and findings (reports) of internal auditors meet my expectations.

\begin{tabular}{|c|l|c|c|c|c|}
\hline \multirow{3}{*}{ Valid } & & Frequency & Percent & Valid Percent & Cumulative Percent \\
\cline { 2 - 6 } & Disagree & 8 & 5.3 & 5.3 & 5.3 \\
\cline { 2 - 6 } & Neutral & 33 & 22.0 & 22.0 & 27.3 \\
\cline { 2 - 6 } & Agree & 62 & 41.3 & 41.3 & 68.7 \\
\cline { 2 - 6 } & Strongly agree & 47 & 31.3 & 31.3 & 100.0 \\
\cline { 2 - 6 } & Total & 150 & 100.0 & 100.0 & \\
\hline
\end{tabular}

The internal auditor reports are highly considered for decision making and internal controls by the management.

\begin{tabular}{|l|l|c|c|c|c|}
\hline & & Frequency & Percent & Valid Percent & Cumulative Percent \\
\hline \multirow{3}{*}{ Valid } & strongly disagree & 4 & 2.7 & 2.7 & 2.7 \\
\cline { 2 - 6 } & Disagree & 13 & 8.7 & 8.7 & 11.3 \\
\cline { 2 - 6 } & Neutral & 31 & 20.7 & 20.7 & 32.0 \\
\cline { 2 - 6 } & Agree & 48 & 32.0 & 32.0 & 64.0 \\
\cline { 2 - 6 } & strongly agree & 54 & 36.0 & 36.0 & 100.0 \\
\cline { 2 - 6 } & Total & 150 & 100.0 & 100.0 & \\
\hline
\end{tabular}

\subsection{Data of questionnaire Administered from Internal auditors' section}

Does your organization/sector have audit committee?

\begin{tabular}{|c|l|c|c|c|c|}
\hline & & Frequency & Percent & Valid Percent & Cumulative Percent \\
\hline \multirow{3}{*}{ Valid } & Yes & 57 & 38.0 & 38.0 & 38.0 \\
\cline { 2 - 6 } & No & 93 & 62.0 & 62.0 & 100.0 \\
\cline { 2 - 6 } & Total & 150 & 100.0 & 100.0 & \\
\hline
\end{tabular}

Indicate your sector

\begin{tabular}{|l|l|c|c|c|c|}
\hline & & Frequency & Percent & Valid Percent & Cumulative Percent \\
\hline \multirow{3}{*}{ Valid } & public business enterprise & 105 & 70.0 & 70.0 & 70.0 \\
\cline { 2 - 6 } & public office/bureau & 45 & 30.0 & 30.0 & 100.0 \\
\cline { 2 - 6 } & Total & 150 & 100.0 & 100.0 & \\
\hline
\end{tabular}

What are the activities carried out by IA in your office/sector?

\begin{tabular}{|c|c|c|c|c|c|}
\hline & & Frequency & Percent & Valid Percent & Cumulative Percent \\
\hline \multirow[t]{6}{*}{ Valid } & compliance audit & 69 & 46.0 & 46.0 & 46.0 \\
\hline & financial audit & 24 & 16.0 & 16.0 & 62.0 \\
\hline & operational/performance audit & 16 & 10.7 & 10.7 & 72.7 \\
\hline & internal control system evaluation & 15 & 10.0 & 10.0 & 82.7 \\
\hline & risk assessment(analysis) audit & 26 & 17.3 & 17.3 & 100.0 \\
\hline & Total & 150 & 100.0 & 100.0 & \\
\hline
\end{tabular}


International Journal of Academic Research in Accounting, Finance and Management Sciences Vol. 10 (1), pp. 25-40, @ 2020 HRMARS (www.hrmars.com)

\subsection{Compliance with IPSAS}

I perform the auditing activities without any interference from anybody and without any influence from the office/sector.

\begin{tabular}{|l|l|c|c|c|c|}
\hline & & Frequency & Percent & Valid Percent & Cumulative Percent \\
\hline \multirow{3}{*}{ Valid } & Disagree & 3 & 2.0 & 2.0 & 2.0 \\
\cline { 2 - 6 } & Neutral & 15 & 10.0 & 10.0 & 12.0 \\
\cline { 2 - 6 } & Agree & 36 & 24.0 & 24.0 & 36.0 \\
\cline { 2 - 6 } & strongly agree & 96 & 64.0 & 64.0 & 100.0 \\
\cline { 2 - 6 } & Total & 150 & 100.0 & 100.0 & \\
\hline
\end{tabular}

I freely decide the scope, time and extent of auditing procedures based on auditing standards and the office/sector policy.

\begin{tabular}{|l|l|c|c|c|c|}
\hline & & Frequency & Percent & Valid Percent & Cumulative Percent \\
\hline \multirow{3}{*}{ Valid } & Neutral & 7 & 4.7 & 4.7 & 4.7 \\
\cline { 2 - 6 } & Agree & 72 & 48.0 & 48.0 & 52.7 \\
\cline { 2 - 6 } & strongly agree & 71 & 47.3 & 47.3 & 100.0 \\
\cline { 2 - 6 } & Total & 150 & 100.0 & 100.0 & \\
\hline
\end{tabular}

I objectively examine auditing issues only meeting on reliable audit evidence and no management interest is involved for adjustment beyond auditing standards and values.

\begin{tabular}{|l|l|c|c|c|c|}
\hline & & Frequency & Percent & Valid Percent & Cumulative Percent \\
\hline \multirow{3}{*}{ Valid } & Disagree & 1 & .7 & .7 & .7 \\
\cline { 2 - 6 } & Neutral & 8 & 5.3 & 5.3 & 6.0 \\
\cline { 2 - 6 } & Agree & 75 & 50.0 & 50.0 & 56.0 \\
\cline { 2 - 6 } & strongly agree & 66 & 44.0 & 44.0 & 100.0 \\
\cline { 2 - 6 } & Total & 150 & 100.0 & 100.0 & \\
\hline
\end{tabular}

I feel free to include any audit finding in my audit work and report directly to responsible body.

\begin{tabular}{|l|l|c|c|c|c|}
\hline \multirow{3}{*}{ Valid } & & Frequency & Percent & Valid Percent & Cumulative Percent \\
\cline { 2 - 6 } & Disagree & 4 & 2.7 & 2.7 & 2.7 \\
\cline { 2 - 6 } & Neutral & 12 & 8.0 & 8.0 & 10.7 \\
\cline { 2 - 6 } & Agree & 105 & 70.0 & 70.0 & 80.7 \\
\cline { 2 - 6 } & strongly agree & 29 & 19.3 & 19.3 & 100.0 \\
\cline { 2 - 6 } & Total & 150 & 100.0 & 100.0 & \\
\hline
\end{tabular}

I can freely access necessary documents, information and data about the organization/sector for my audit work.

\begin{tabular}{|l|l|c|c|c|c|}
\hline & & Frequency & Percent & Valid Percent & Cumulative Percent \\
\hline \multirow{3}{*}{ Valid } & strongly disagree & 3 & 2.0 & 2.0 & 2.0 \\
\cline { 2 - 6 } & Disagree & 2 & 1.3 & 1.3 & 3.3 \\
\cline { 2 - 6 } & Neutral & 14 & 9.3 & 9.3 & 12.7 \\
\cline { 2 - 6 } & Agree & 77 & 51.3 & 51.3 & 64.0 \\
\cline { 2 - 6 } & strongly agree & 54 & 36.0 & 36.0 & 100.0 \\
\cline { 2 - 6 } & Total & 150 & 100.0 & 100.0 & \\
\hline
\end{tabular}




\subsection{Audit Mechanism}

Dependent variable: Effectiveness of public sector financial audit Factor: Audit Mechanism

\section{ANOVA}

Effectiveness of public sector financial audit

\begin{tabular}{|l|c|c|c|c|c|}
\hline & Sum of Squares & df & Mean Square & F & Sig. \\
\hline Between Groups & 8.697 & 2 & 4.348 & 12.589 & .000 \\
\hline Within Groups & 50.776 & 147 & .345 & & \\
\hline Total & 59.473 & 149 & & & \\
\hline
\end{tabular}

The office/sector has enough awareness and good perception about internal auditing roles.

\begin{tabular}{|l|l|c|c|c|c|}
\hline & & Frequency & Percent & Valid Percent & Cumulative Percent \\
\hline \multirow{3}{*}{ Valid } & Neutral & 18 & 12.0 & 12.0 & 12.0 \\
\cline { 2 - 6 } & Agree & 73 & 48.7 & 48.7 & 60.7 \\
\cline { 2 - 6 } & strongly agree & 59 & 39.3 & 39.3 & 100.0 \\
\cline { 2 - 6 } & Total & 150 & 100.0 & 100.0 & \\
\hline
\end{tabular}

The office/sector considers internal auditing practices as a value adding activity and I work smoothly and regularly with the management.

\begin{tabular}{|l|l|c|c|c|c|}
\hline \multirow{3}{*}{ Valid } & & Frequency & Percent & Valid Percent & Cumulative Percent \\
\cline { 2 - 6 } & Disagree & 1 & .7 & .7 & .7 \\
\cline { 2 - 6 } & Neutral & 34 & 22.7 & 22.7 & 23.3 \\
\cline { 2 - 6 } & Agree & 75 & 50.0 & 50.0 & 73.3 \\
\cline { 2 - 6 } & strongly agree & 40 & 26.7 & 26.7 & 100.0 \\
\cline { 2 - 6 } & Total & 150 & 100.0 & 100.0 & \\
\hline
\end{tabular}

The office/sector encourages me to implement my recommendations, to promote value added activities and to strength internal audit function.

\begin{tabular}{|l|l|c|c|c|c|}
\hline & & Frequency & Percent & Valid Percent & Cumulative Percent \\
\hline \multirow{3}{*}{ Valid } & strongly disagree & 1 & .7 & .7 & .7 \\
\cline { 2 - 6 } & Disagree & 1 & .7 & .7 & 1.3 \\
\cline { 2 - 6 } & Neutral & 41 & 27.3 & 27.3 & 28.7 \\
\cline { 2 - 6 } & Agree & 65 & 43.3 & 43.3 & 72.0 \\
\cline { 2 - 6 } & strongly agree & 42 & 28.0 & 28.0 & 100.0 \\
\cline { 2 - 6 } & Total & 150 & 100.0 & 100.0 & \\
\hline
\end{tabular}

The position/status of internal audits is clearly recognized by the management in my office/sector.

\begin{tabular}{|l|l|c|c|c|c|}
\hline \multirow{3}{*}{ Valid } & & Frequency & Percent & Valid Percent & Cumulative Percent \\
\cline { 2 - 6 } & Disagree & 3 & 2.0 & 2.0 & 2.0 \\
\cline { 2 - 6 } & Neutral & 35 & 23.3 & 23.3 & 25.3 \\
\cline { 2 - 6 } & Agree & 75 & 50.0 & 50.0 & 75.3 \\
\cline { 2 - 6 } & Strongly agree & 37 & 24.7 & 24.7 & 100.0 \\
\cline { 2 - 6 } & Total & 150 & 100.0 & 100.0 & \\
\hline
\end{tabular}


International Journal of Academic Research in Accounting, Finance and Management Sciences Vol. 10 (1), pp. 25-40, @ 2020 HRMARS (www.hrmars.com)

\subsection{Mechanism of Financial Regulatory Body}

Dependent variable: Effectiveness of public sector financial audit

Factor: Mechanism of Financial Regulatory Body

\section{ANOVA}

Effectiveness of public sector financial audit

\begin{tabular}{|l|c|c|c|c|c|}
\hline & Sum of Squares & df & Mean Square & F & Sig. \\
\hline Between Groups & .666 & 2 & .333 & .832 & .437 \\
\hline Within Groups & 58.808 & 147 & .400 & & \\
\hline Total & 59.473 & 149 & & & \\
\hline
\end{tabular}

Internal audit charter is maintained (available) in my office/sector.

\begin{tabular}{|l|l|c|c|c|c|}
\hline & & Frequency & Percent & Valid Percent & Cumulative Percent \\
\hline \multirow{3}{*}{ Valid } & strongly disagree & 2 & 1.3 & 1.3 & 1.3 \\
\cline { 2 - 6 } & Disagree & 1 & .7 & .7 & 2.0 \\
\cline { 2 - 6 } & Neutral & 6 & 4.0 & 4.0 & 6.0 \\
\cline { 2 - 6 } & Agree & 38 & 25.3 & 25.3 & 31.3 \\
\cline { 2 - 6 } & strongly agree & 103 & 68.7 & 68.7 & 100.0 \\
\cline { 2 - 6 } & Total & 150 & 100.0 & 100.0 & \\
\hline
\end{tabular}

The purpose and authority of internal audit is clearly defined in charter.

\begin{tabular}{|l|l|c|c|c|c|}
\hline & & Frequency & Percent & Valid Percent & Cumulative Percent \\
\hline \multirow{3}{*}{ Valid } & strongly disagree & 1 & .7 & .7 & .7 \\
\cline { 2 - 6 } & Disagree & 1 & .7 & .7 & 1.3 \\
\cline { 2 - 6 } & Neutral & 3 & 2.0 & 2.0 & 3.3 \\
\cline { 2 - 6 } & Agree & 52 & 34.7 & 34.7 & 38.0 \\
\cline { 2 - 6 } & strongly agree & 93 & 62.0 & 62.0 & 100.0 \\
\cline { 2 - 6 } & Total & 150 & 100.0 & 100.0 & \\
\hline
\end{tabular}

The purpose and authority of internal audit charter is in line with "standard for the professional practice" formulated by the institute of internal auditors.

\begin{tabular}{|l|l|c|c|c|c|}
\hline & & Frequency & Percent & Valid Percent & Cumulative Percent \\
\hline \multirow{3}{*}{ Valid } & Disagree & 3 & 2.0 & 2.0 & 2.0 \\
\cline { 2 - 6 } & Neutral & 8 & 5.3 & 5.3 & 7.3 \\
\cline { 2 - 6 } & Agree & 78 & 52.0 & 52.0 & 59.3 \\
\cline { 2 - 6 } & strongly agree & 61 & 40.7 & 40.7 & 100.0 \\
\cline { 2 - 6 } & Total & 150 & 100.0 & 100.0 & \\
\hline
\end{tabular}

The IA charter specifies the internal audit activity's position within the office; authorized access to records, personnel, and physical properties relevant to the performance of engagements, and the define scope of IA activities.

\begin{tabular}{|l|l|c|c|c|c|}
\hline \multirow{3}{*}{ Valid } & & Frequency & Percent & Valid Percent & Cumulative Percent \\
\cline { 2 - 6 } & Disagree & 2 & 1.3 & 1.3 & 1.3 \\
\cline { 2 - 6 } & Neutral & 9 & 6.0 & 6.0 & 7.3 \\
\cline { 2 - 6 } & Agree & 94 & 62.7 & 62.7 & 70.0 \\
\cline { 2 - 6 } & Strongly agree & 45 & 30.0 & 30.0 & 100.0 \\
\cline { 2 - 6 } & Total & 150 & 100.0 & 100.0 & \\
\hline
\end{tabular}


Punishments for violations of inter audit procedures at different levels are adequate?

\begin{tabular}{|l|l|c|c|c|c|}
\hline & & Frequency & Percent & Valid Percent & Cumulative Percent \\
\hline \multirow{4}{*}{ Valid } & Disagree & 4 & 2.7 & 2.7 & 2.7 \\
\cline { 2 - 6 } & Neutral & 18 & 12.0 & 12.0 & 14.7 \\
\cline { 2 - 6 } & Agree & 80 & 53.3 & 53.3 & 68.0 \\
\cline { 2 - 6 } & strongly agree & 48 & 32.0 & 32.0 & 100.0 \\
\cline { 2 - 6 } & Total & 150 & 100.0 & 100.0 & \\
\hline
\end{tabular}

\subsection{Technical Qualification of Audit Stuffs}

Dependent variable: Effectiveness of public sector financial audit Factor: Technical Qualification of Audit Stuffs

\section{ANOVA}

Effectiveness of public sector financial audit

\begin{tabular}{|l|c|c|c|c|c|}
\hline & Sum of Squares & df & Mean Square & F & Sig. \\
\hline Between Groups & 1.452 & 2 & .726 & 1.839 & .163 \\
\hline Within Groups & 58.021 & 147 & .395 & & \\
\hline Total & 59.473 & 149 & & & \\
\hline
\end{tabular}

I receive full cooperation, access to records and information from my office/sector.

\begin{tabular}{|l|l|c|c|c|c|}
\hline & & Frequency & Percent & Valid Percent & Cumulative Percent \\
\hline \multirow{3}{*}{ Valid } & Disagree & 1 & .7 & .7 & .7 \\
\cline { 2 - 6 } & Neutral & 19 & 12.7 & 12.7 & 13.3 \\
\cline { 2 - 6 } & Agree & 61 & 40.7 & 40.7 & 54.0 \\
\cline { 2 - 6 } & Strongly agree & 69 & 46.0 & 46.0 & 100.0 \\
\cline { 2 - 6 } & Total & 150 & 100.0 & 100.0 & \\
\hline
\end{tabular}

I can get the necessary resources (facilities) that help me to perform auditing activities as needed.

\begin{tabular}{|l|l|c|c|c|c|}
\hline & & Frequency & Percent & Valid Percent & Cumulative Percent \\
\hline \multirow{3}{*}{ Valid } & Disagree & 2 & 1.3 & 1.3 & 1.3 \\
\cline { 2 - 6 } & Neutral & 21 & 14.0 & 14.0 & 15.3 \\
\cline { 2 - 6 } & Agree & 92 & 61.3 & 61.3 & 76.7 \\
\cline { 2 - 6 } & strongly agree & 35 & 23.3 & 23.3 & 100.0 \\
\cline { 2 - 6 } & Total & 150 & 100.0 & 100.0 & \\
\hline
\end{tabular}

The office/sector supports me by providing training in order to improve my skill and update with the field.

\begin{tabular}{|l|l|c|c|c|c|}
\hline & & Frequency & Percent & Valid Percent & Cumulative Percent \\
\hline \multirow{4}{*}{ Valid } & Disagree & 2 & 1.3 & 1.3 & 1.3 \\
\cline { 2 - 6 } & Neutral & 25 & 16.7 & 16.7 & 18.0 \\
\cline { 2 - 6 } & Agree & 69 & 46.0 & 46.0 & 64.0 \\
\cline { 2 - 6 } & strongly agree & 54 & 36.0 & 36.0 & 100.0 \\
\cline { 2 - 6 } & Total & 150 & 100.0 & 100.0 & \\
\hline
\end{tabular}

The office/sector supports me to introduce myself with new technology, policy or procedures when it is necessary.

\begin{tabular}{|c|l|c|c|c|c|}
\hline & & Frequency & Percent & Valid Percent & Cumulative Percent \\
\hline \multirow{2}{*}{ Valid } & Disagree & 3 & 2.0 & 2.0 & 2.0 \\
\cline { 2 - 7 } & Neutral & 29 & 19.3 & 19.3 & 21.3 \\
\hline
\end{tabular}




\begin{tabular}{|l|l|c|c|c|c|}
\hline \multirow{2}{*}{} & Agree & 77 & 51.3 & 51.3 & 72.7 \\
\cline { 2 - 6 } & strongly agree & 41 & 27.3 & 27.3 & 100.0 \\
\cline { 2 - 5 } & Total & 150 & 100.0 & 100.0 & \\
\hline
\end{tabular}

The office/sector supports internal auditing staffs by budgeting funds for certification to have relevant education in auditing that allows them to audit all the organizations/sectors systems.

\begin{tabular}{|l|l|c|c|c|c|}
\hline & & Frequency & Percent & Valid Percent & Cumulative Percent \\
\hline \multirow{4}{*}{ Valid } & strongly disagree & 1 & .7 & .7 & .7 \\
\cline { 2 - 6 } & Disagree & 13 & 8.7 & 8.7 & 9.3 \\
\cline { 2 - 6 } & Neutral & 23 & 15.3 & 15.3 & 24.7 \\
\cline { 2 - 6 } & Agree & 58 & 38.7 & 38.7 & 63.3 \\
\cline { 2 - 6 } & strongly agree & 55 & 36.7 & 36.7 & 100.0 \\
\cline { 2 - 6 } & Total & 150 & 100.0 & 100.0 & \\
\hline
\end{tabular}

\section{Regression Analysis}

\section{Model Summary}

\begin{tabular}{|c|c|c|c|c|}
\hline Model & $\mathbf{R}$ & R Square & Adjusted R Square & Std. Error of the Estimate \\
\hline 1 & $.415^{\mathrm{a}}$ & .172 & .150 & .24330 \\
\hline
\end{tabular}

a. Predictors: (Constant), Technical Qualification of Audit Stuffs, Compliance with IPSAS, Mechanism of Financial Regulatory Body, Audit Mechanism.

The value of $R$ square is equal to regression sum of squares divided by total sum of squares. Adjusted $\mathrm{R}$ square is depending on sample size. Here, $15 \%$ of the variance in depressive symptoms can be explained by one's rejection sensitivity. The value of Std. Error of the Estimate is depending on the value of mean square.

The $R$ value represents the simple correlation and is $.415^{\text {a }}$ (the "R" Column), which indicates a high degree of correlation. The $R^{2}$ value (the "R Square" column) indicates how much of the total variation in the dependent variable, can be explained by the independent variable. The $R^{2}$ value of .172 which represents $17.2 \%$ indicates low variation in the dependent variable

\section{ANOVA}

\begin{tabular}{|l|l|c|c|c|c|c|}
\hline \multicolumn{2}{|c|}{ Model } & Sum of Squares & df & Mean Square & F & Sig. \\
\hline \multirow{2}{*}{1} & Regression & 1.788 & 4 & .447 & 7.549 & $.000^{\mathrm{a}}$ \\
\cline { 2 - 8 } & Residual & 8.583 & 145 & .059 & & \\
\cline { 2 - 7 } & Total & 10.371 & 149 & & & \\
\hline
\end{tabular}

a. Predictors: (Constant), Technical Qualification of Audit Stuffs, Compliance with IPSAS, Mechanism of Financial Regulatory Body, Audit Mechanism

b. Dependent Variable: Effectiveness of Internal auditors

This ANOVA indicates that the regression model predicts the dependent variable significantly well. The "Sig." column which indicates the statistical significance of the regression model that was run. Here, the value is 0.000 , which is less than $p<0.05$, and indicates that, overall, the regression model statistically significantly predicts the outcome variable (i.e., it is a good fit for the data).

\section{Coefficients $^{\mathrm{a}}$}

\begin{tabular}{|c|c|c|c|c|c|c|}
\hline \multirow{2}{*}{\multicolumn{2}{|c|}{ Model }} & \multicolumn{2}{|c|}{$\begin{array}{l}\text { Unstandardized } \\
\text { Coefficients }\end{array}$} & \multirow{2}{*}{$\begin{array}{c}\begin{array}{c}\text { Standardized } \\
\text { Coefficients }\end{array} \\
\text { Beta }\end{array}$} & \multirow[t]{2}{*}{$\mathbf{t}$} & \multirow[t]{2}{*}{ Sig. } \\
\hline & & B & Std. Error & & & \\
\hline \multirow[t]{2}{*}{1} & (Constant) & 2.718 & .309 & & 8.809 & .000 \\
\hline & Compliance with IPSAS & .061 & .053 & .088 & 1.146 & .254 \\
\hline
\end{tabular}




\begin{tabular}{|l|l|c|c|c|c|c|}
\hline \multirow{2}{*}{ Audit Mechanism } & .143 & .045 & .262 & 3.147 & .002 \\
\cline { 2 - 7 } & Mechanism of Financial Regulatory Body & .088 & .048 & .148 & 1.831 & .069 \\
\cline { 2 - 7 } & Technical Qualification of Audit Stuffs & .058 & .047 & .106 & 1.219 & .225 \\
\hline
\end{tabular}

a. Dependent Variable: Effectiveness of Internal auditors

Here $\mathrm{R}$ is the regression coefficient and the regression parameter $\mathrm{B}$ which we have to interpret. We consider Effectiveness of internal auditors as dependent variable and we have four independent variables. Here the value $B$ are the 2.718 (intercept), 0.061 ( $1^{\text {st }}$ regression coefficient), $0.143\left(2^{\text {nd }}\right.$ regression coefficient), 0.088 ( ${ }^{\text {rd }}$ regression coefficient) and 0.058 (last regression coefficient).

One unit increase of $1^{\text {st }}$ (Compliance with IPSAS) regression coefficient when other coefficients are remaining constant then the average increase of dependent variable (Effectiveness of Internal auditors) is 2.718 unit.

One unit increase of 2 nd (Audit Mechanism) regression coefficient when other coefficients are remain constant then the average increase of dependent variable (Effectiveness of Internal auditors) is 2.718 unit.

One unit increase of 3rd (Mechanism of Financial Regulatory Body) regression coefficient when other coefficients are remaining constant then the average increase of dependent variable (Effectiveness of Internal auditors) is 2.718 unit.

One unit increase of 4th (Technical Qualification of Audit Stuffs) regression coefficient when other coefficients are remaining constant then the average increase of dependent variable(Effectiveness of Internal auditors) is 2.718 unit.

As detailed above there is a strong conclusion that "positive relationship exists between independent variables which are (Compliance with IPSAS, Audit Mechanism, Mechanism of Financial Regulatory Body, Technical Qualification of Audit Stuffs) and the dependent variable which is the Effectiveness of Internal auditors".

Correlations Analysis

\begin{tabular}{|c|c|c|c|c|c|c|}
\hline & & $\begin{array}{c}\text { Effectiveness } \\
\text { of Internal } \\
\text { auditors }\end{array}$ & $\begin{array}{l}\text { Compliance } \\
\text { with IPSAS }\end{array}$ & $\begin{array}{c}\text { Audit } \\
\text { Mechanism }\end{array}$ & $\begin{array}{c}\text { Risk } \\
\text { management } \\
\text { and control }\end{array}$ & $\begin{array}{c}\text { Technical } \\
\text { Qualification of } \\
\text { Audit Stuffs }\end{array}$ \\
\hline \multirow{3}{*}{$\begin{array}{l}\text { Effectiveness of Internal } \\
\text { auditors }\end{array}$} & Pearson Correlation & 1 & .155 & $.352^{* *}$ & $.251^{* *}$ & $.277^{* *}$ \\
\hline & Sig. (2-tailed) & & .059 & .000 & .002 & .001 \\
\hline & $N$ & 150 & 150 & 150 & 150 & 150 \\
\hline \multirow[t]{3}{*}{ Compliance with IPSAS } & Pearson Correlation & .155 & 1 & .151 & .062 & $.167^{*}$ \\
\hline & Sig. (2-tailed) & .059 & & .066 & .450 & .041 \\
\hline & $N$ & 150 & 150 & 150 & 150 & 150 \\
\hline \multirow[t]{3}{*}{ Audit Mechanism } & Pearson Correlation & $.352^{* *}$ & .151 & 1 & $.232^{* *}$ & $.400^{* *}$ \\
\hline & Sig. (2-tailed) & .000 & .066 & & .004 & .000 \\
\hline & N & 150 & 150 & 150 & 150 & 150 \\
\hline \multirow{3}{*}{$\begin{array}{l}\text { Mechanism of Financial } \\
\text { Regulatory Body }\end{array}$} & Pearson Correlation & $.251^{* *}$ & .062 & $.232^{* *}$ & 1 & $.348^{* *}$ \\
\hline & Sig. (2-tailed) & .002 & .450 & .004 & & .000 \\
\hline & $\mathrm{N}$ & 150 & 150 & 150 & 150 & 150 \\
\hline \multirow{3}{*}{$\begin{array}{l}\text { Technical Qualification of } \\
\text { Audit Stuffs }\end{array}$} & Pearson Correlation & $.277^{* *}$ & $.167^{*}$ & $.400^{* *}$ & $.348^{* *}$ & 1 \\
\hline & Sig. (2-tailed) & .001 & .041 & .000 & .000 & \\
\hline & N & 150 & 150 & 150 & 150 & 150 \\
\hline \multicolumn{7}{|c|}{ **. Correlation is significant at the 0.01 level (2-tailed). } \\
\hline \multicolumn{4}{|c|}{ *. Correlation is significant at the 0.05 level (2-tailed). } & & & \\
\hline
\end{tabular}

From above table we can see the value of correlation coefficient $r$. we know the values of $r$ lies between -1 to 1 . If the value is close to 1 then there exists a strong relationship between the variables. And 
also, if the values of $r$ close to zero then there exist weak relation between the variables. If the value of $r$ is negative then there exists native relation between the variables.

From above table:

The relationship between Effectiveness of Internal auditors and Compliance with IPSAS is approximately good because the value of the coefficient is 0.155 .

The relationship between Effectiveness of Internal auditors and Audit Mechanism is approximately good because the value of the coefficient is 0.352 .

The relationship between Effectiveness of Internal auditors and Mechanism of Financial Regulatory Body is approximately good because the value of the coefficient is 0.277 .

The relationship between Compliance with IPSAS and Audit Mechanism is approximately good because the value of the coefficient is 0.151 .

The relationship between Compliance with IPSAS and Mechanism of Financial Regulatory Body is approximately good because the value of the coefficient is 0.062 .

The relationship between Compliance with IPSAS and Technical Qualification of Audit Stuffs is approximately good because the value of the coefficient is 0.167 .

The relationship between Audit Mechanism and Mechanism of Financial Regulatory Body is approximately good because the value of the coefficient is 0.232 .

The relationship between Audit Mechanism and Technical Qualification of Audit Stuffs is approximately good because the value of the coefficient is 0.40 .

T-test:

The t-test (also called Student's t-test) is one of many statistical significance tests that compares two supposedly equal amounts of data to see if they are really the same or not. The t-test helps researchers to determine if a hypothesis is supported or not.

Table 1. 1 One sample test

One-Sample Statistics

\begin{tabular}{|l|c|c|c|c|}
\hline \multicolumn{1}{|c|}{} & $\mathbf{N}$ & Mean & Std. Deviation & Std. Error Mean \\
\hline Audit Mechanism & 150 & 2.78 & .542 & .044 \\
\hline Mechanism of Financial Regulatory Body & 150 & 2.90 & .397 & .032 \\
\hline Technical Qualification of Audit Stuffs & 150 & 2.80 & .543 & .044 \\
\hline Effectiveness of public sector financial audit & 150 & 2.51 & .632 & .052 \\
\hline Compliance with IPSAS & 150 & 2.40 & .760 & .062 \\
\hline
\end{tabular}

\section{One-Sample Test}

\begin{tabular}{|c|c|c|c|c|c|c|}
\hline & \multicolumn{6}{|c|}{ Test Value $=0$} \\
\hline & \multirow[t]{2}{*}{$\mathbf{t}$} & \multirow[t]{2}{*}{ df } & \multirow[t]{2}{*}{ Sig. (2-tailed) } & \multirow{2}{*}{$\begin{array}{c}\text { Mean } \\
\text { Difference }\end{array}$} & \multicolumn{2}{|c|}{$\begin{array}{l}\text { 95\% Confidence Interval } \\
\text { of the Difference }\end{array}$} \\
\hline & & & & & Lower & Upper \\
\hline Audit Mechanism & 62.841 & 149 & .000 & 2.780 & 2.69 & 2.87 \\
\hline Mechanism of Financial Regulatory Body & 89.434 & 149 & .000 & 2.900 & 2.84 & 2.96 \\
\hline Technical Qualification of Audit Stuffs & 63.106 & 149 & .000 & 2.800 & 2.71 & 2.89 \\
\hline $\begin{array}{l}\text { Effectiveness of public sector financial } \\
\text { audit }\end{array}$ & 48.722 & 149 & .000 & 2.513 & 2.41 & 2.62 \\
\hline Compliance with IPSAS & 38.690 & 149 & .000 & 2.400 & 2.28 & 2.52 \\
\hline
\end{tabular}

If the value of sig. ( $p$-value) less than .05 , then the model will be significant. Here in the model the value of sig. is .00 . So, the model is significant. 


\section{Conclusions}

More accurate and timely information about public sector unit spending, performance and quality is needed. Increasing productivity without affecting the quality of services requires a better understanding of how it uses resources in the delivery of services. The financial management of the public sector is a matter of public economic agenda, which is free from irregularities in the public sector, to carefully manage the dreaded assets and taxes contained in the central bank's consolidated funds to provide private services (Othman et al., 2015).

There is a need for a more integrated and integrated approach across the public sector. This is necessary to improve productivity and to protect the quality of service delivery systems that require jointup work, for example in health and social care. Better coordination allows for better sharing of good practice and facilitating the work of the whole system more effectively.

The weaknesses of internal control systems in public sector resources and financial management are a complex field that is currently lacking attention due to the continued neglect of regulatory elements, so large public financial irregularities in the management, allocation, and accounting of state assets are under public control.

This study is exceptionally crucial to demonstrate the adequacy of the internal audit by surveying the elements which decides the public sectors by inner review adequacy. And also, this study is vital for the exact proofs for the public population part workplaces to look at their approaches and methodology, and for the future researchers additionally critical to build up an applied writing advancement. The auditors have a noteworthy effect to control any resistance action in accordance with the built up arrangements, arranges, strategies, laws and controls, set by the management which could significantly affect the institute's operations. The auditors are likewise crucial to increase the value of the organization, to stay away from disappointment and to spare the working and organization costs as per the association's approaches and methodology.

\section{Recommendations in alignment with the research objectives}

The specific objective of this study is,

- To investigate the public sector financial audit effectiveness in the Abu Dhabi Police (ADP), UAE.

- Other objectives are,

- To investigate impact of infrastructure for financial audit in the Police Department to improve performance

- To investigate the competency of the public sector financial auditors in the ADP, UAE

The following recommendations can be listed from all the analysis for effectiveness of public sector financial audit: a comprehensive study on the police department of Abu Dhabi, UAE.

Firstly, the public sector financial audit should not be only for statutory requirement but for internal control enhancement as well.

Secondly, the public sector financial audit should not be only annually or interim, there should be the scope of surprise audit as well.

Thirdly, segregation of duties is essential for a proper internal control on the activities in alignment with public sector financial audit.

Fourthly, introduction of more key personnel with sound knowledge of accounting, external and internal auditing to support the growth of the infrastructure of the accounts department of the ministry of interior.

Fifthly, there needs to be more transparency in the books of the final accounts disclosure requirement so as to make the books of financial audited statement more acceptable.

Sixthly, e financial audit reports to be made more available for ensuring good corporate governance.

Finally, more involvement of independent auditor not locally but globally recognized audit firm as well in addition to provision of joint audit as well. 


\section{Contribution}

Well, after examining the effectiveness of public sector financial audit: a comprehensive study on the police department of Abu Dhabi, UAE. - the first objective of the study, it is seen that the effectiveness of the public sector financial audit will be more appropriate if there is a scope of joint audit and both local and global reputed audit firms are involved. The research has focused the need of such need of the hour independent acceptability to be at par with leading nation globally.

Impact of the competency of the public sector financial audit was observed to be very ineffective due to the fact it is just a routine check, rather the competency would have been improved if in addition surprise public sector financial audit was determined and put into place. The researchers are looking for more contributions in the arena and by next few months a comprehensive contribution can be highlighted.

\section{Limitation of the research}

The following limitation was observed by the researcher.

Firstly, the collection of data had an issue as many employees were not having the required information on financial matters due to its confidentiality. Secondly, time constraints. Finally, most of the data are very classified and used here very cautiously.

\section{References}

1. Mansour, A. M. (2017). Has The United Arab Emirates Federal Government Succeeded To Transform Its Federal Bureaucracy Into A New Public Management System?, 18(1), 119.

2. Fan, J. \& Wong, T. J. (2005). Do external auditors perform a corporate governance role in emerging markets? Evidence from East Asia. Journal of Accounting Research, 43(1), 35-72.

3. Jill, M. D., \& Houmes, R. (2014). COSO's Updated Internal Control and Enterprise Risk Management Frameworks. The CPA Journal, 84(5), 54.

4. Grau-Gumbau, R., Agut-Nieto, S., Llorens-Gumbau, S., Martinez-Martinez, I. N. (2002). Managerial training needs: A study of Spanish tourism organization. Tourism \& Hospitality Research, Feb2002, 3(3), 234-244.

5. Güvemli, B., Yildiz, F. (2010) "Commercial Books Under Turkish Commercial Laws Concerning Accounting", Pecvnia, Revista de la Facultad de Ciencias Económicas y Empresariales de la Universidad de León, 85-104, no.11.

6. Tricker, R. B., \& Tricker, R. I. (2015). Corporate governance: Principles, policies, and practices. Oxford University Press, USA

7. Yuan, D., Hua, Z. \& Junxi, Z. (2007). Private vs. state ownership and earnings management: evidence from Chinese listed companies. Corporate Governance: An International Review, 15(2), 223-238. 